\title{
Cosmologie et physique des particules : questions croisées
}

Francis Bernardeau $^{(1)}$ (francis.bernardeau@iap.fr) et Christophe Grojean ${ }^{(2)}$ (christophe.grojean@cern.ch) (1) Institut d'Astrophysique de Paris, UMR 7095 de l'Université Pierre et Marie Curie et du CNRS, 98 bis boulevard Arago, 75014 Paris

(2) Institució Catalana de recerca i estudis avançats, Institut de Física d’Altes Energies, Universitat Autònoma de Barcelona, 08193 Bellaterra, Barcelone, Espagne

\section{La physique est entrée}

dans son histoire moderne avec la chute d'une pomme sur la tête de Newton.

Depuis, notre connaissance des lois fondamentales de notre monde n'a cessé de s'enrichir de l'étude de l'infiniment grand et de I'infiniment petit. Aujourd'hui encore, le dialogue perdure entre ces deux infinis.

Dans cet article, deux physiciens théoriciens, un astrophysicien (FB) et un physicien des particules (CG), décrivent le développement commun de leurs disciplines à travers les réponses apportées à sept questions clés.

Cet article est dédié

à la mémoire d'André Morel.

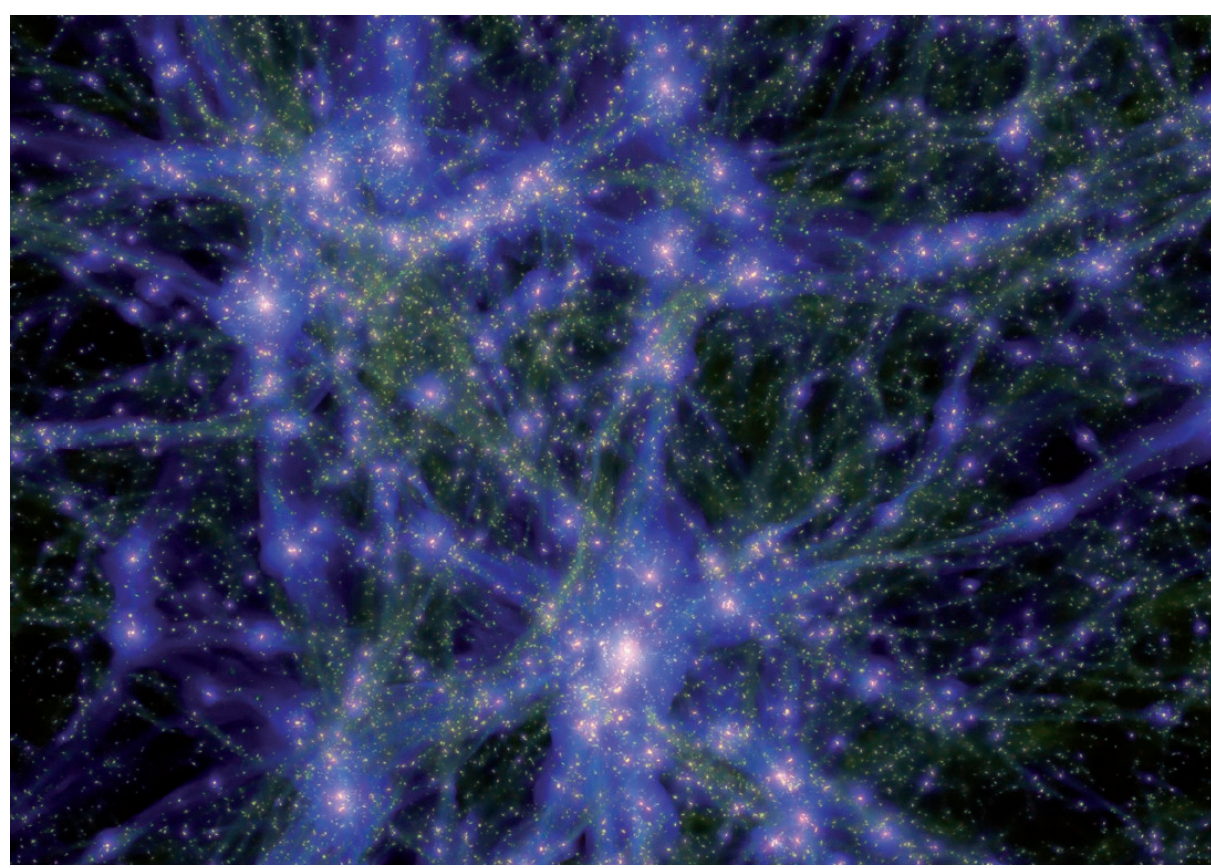

Image composite d'une simulation cosmologique haute résolution, montrant la répartition de matière noire et de matière baryonique dans l'univers. En rouge : densité de matière noire ; en vert : densité de matière baryonique ; en bleu : température du gaz. (Projet Horizon, voir www.projet-horizon.fr).

\section{Qu'y a-t-il de commun} entre la cosmologie et la physique des particules?

Les pâtissiers, et tous les bons gourmands, savent qu'un kilogramme de sucre n'est que la somme des masses des petits grains de sucre qui le composent. D'autres sauront certainement que ces grains de sucre euxmêmes ne sont que des assemblages de molécules composées d'atomes de carbone, d'oxygène et d'hydrogène liés entre eux par des forces électromagnétiques. Lorsqu'on chauffe le sucre, on confere de l'énergie cinétique à ces atomes qui deviennent vite assez énergétiques pour casser les liaisons chimiques et ainsi se libérer de leurs voisins et éventuellement se réorganiser pour former du caramel.
Ce qui se passe dans une casserole s'est d'abord déroulé dans l'univers dans son ensemble au cours de son histoire et des différentes périodes de "caramélisation " (appelées " transitions de phase " par les scientifiques) qu'il a connues (voir encadré 1). Plus on remonte l'histoire de l'univers, plus il était dense et chaud, conférant aux grains de matière suffisamment d'énergie pour s'affranchir d'abord des liaisons électromagnétiques puis des forces de cohésion associées aux interactions faibles et fortes, et ainsi révéler les briques élémentaires de la matière : les quarks, dont sont constitués les protons et les neutrons qui forment les noyaux des atomes, et les leptons, comme l'électron qui orbite autour des noyaux. Le comportement de ces particules élémentaires est régi par les lois de la relativité et de la 


\section{La construction du modèle concordant de cosmologie}

La relativité générale constitue le socle théorique sur lequel est bâtie la cosmologie moderne. Les solutions d'espace en expansion ont été identifiées dès les années 1920, puis, après la découverte de l'expansion de l'univers par G. Lemaître en 1927 et E. Hubble en 1929 (fig. E1), les mécanismes fondamentaux menant à l'émergence des grandes structures de l'univers - par instabilités gravitationnelles de petites fluctuations de métrique - ont été identifiés. Pourtant, il faudra attendre les années 1960 et la découverte par A. Penzias et R. Wilson d'un fond diffus micro-onde à $3 \mathrm{~K}$ en 1965, pour que s'ouvre une nouvelle ère où les détails du modèle dit du Big-Bang chaud ont pu être précisés petit à petit.

À la base de ce paradigme est l'idée que I'univers est en expansion et donc en constant refroidissement. Tels des paléontologues, les cosmologistes sont amenés à rechercher I'origine de l'ensemble des objets qui relèvent de l'astrophysique (des planètes aux galaxies) ou de la physique des hautes énergies (des particules élémentaires aux noyaux lourds). Ce programme de recherche est loin d'être achevé.

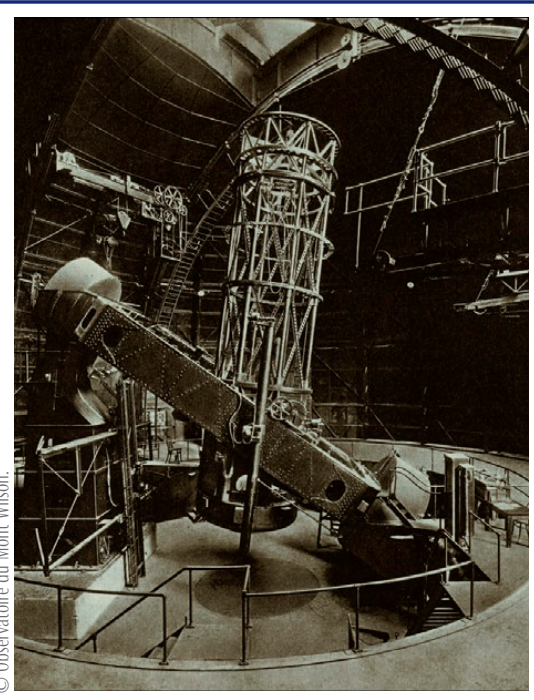

E1. Télescope Hooker de 2,5 m du Mont Wilson, avec lequel E. Hubble mesura les premières vitesses de récession de nébuleuses (galaxies), qui ont permis à G. Lemaître en 1927 (Annales de la Société Scientifique de Bruxelles, A47, pp. 49-59), puis à E. Hubble en 1929, d'établir la loi qui porte le nom de ce dernier.

Les cosmologistes peuvent cependant s'enorgueillir de nombreux succès :

- Les ères et sites de formation des noyaux ont été identifiés. La nucléosynthèse primordiale prédit de manière précise l'abondance des noyaux légers, notamment l'hélium, au travers d'une cinétique chimique hors d'équilibre contrôlée par le taux d'expansion de l'univers, tandis que les noyaux lourds se forment par nucléosynthèse explosive dans les supernovæ.

- Le mécanisme d'instabilité gravitationnelle au sein d'un univers en expansion est à l'origine des grandes structures de l'univers, formées à partir de fluctuations initiales de la géométrie d'espace-temps. Ce mécanisme détermine aussi bien les flots de vitesse à grande échelle que l'amplitude des distorsions gravitationnelles (effets de lentille) observées maintenant couramment dans les catalogues profonds de galaxies. C'est ce mécanisme qui donne le tempo pour la formation des premières galaxies, des premières étoiles, éventuellement de trous noirs, etc., résultant du jeu croisé entre les différentes composantes de l'univers.

- Enfin le fond diffus cosmologique, avec ses propriétés spectrales, l'amplitude de ses anisotropies de température et de polarisation, est la véritable pierre de Rosette de la cosmologie moderne. Il est la surface (interne) de l'univers qui représente le lieu, et de manière équivalente le moment, où l'univers devient transparent lorsque I'agitation thermique décroît suffisamment pour permettre aux électrons de se lier aux noyaux - on parle de recombinaison. On y voit littéralement à l'œuvre le refroidissement d'un plasma chaud; on y lit ses modes propres de vibration; on y mesure sa composition et ses propriétés thermodynamiques.

Le modèle cosmologique, dit concordant, qui précise le contenu en matière et en énergie de I'univers, reprend ces découvertes dans un cadre théorique unique. II introduit cependant un certain nombre d'ingrédients qui n'ont pas (encore) été identifiés du côté de la physique des particules. Notamment la matière noire, plus justement une matière transparente sans interaction électromagnétique, dont l'existence se révèle au moment de la recombinaison entre électrons et noyaux et à toutes les échelles cosmologiques. Le modèle concordant s'appuie aussi, sans que ce paradigme soit parfaitement justifié, sur l'existence de fluctuations de métriques primordiales, dont l'inflation (voir question 5, p. 12) serait à l'origine. Enfin, le bilan à la fois dynamique et géométrique montre qu'on ne peut rendre compte de la dynamique d'expansion sans faire appel à une constante cosmologique, ou à une composante d'énergie " noire ", de densité de l'ordre de $10^{-15} \mathrm{~J} / \mathrm{cm}^{3}$, dont la nature même est très difficile à expliquer du point de vue de la physique des hautes énergies.

$>>$

mécanique quantique, qui permettent de convertir l'énergie cinétique des collisions en masse, donc en matière. Ainsi apparaissent de nouvelles particules, souvent bien différentes de celles qui forment les atomes d'aujourd'hui. C'est ainsi que l'univers, en se réchauffant, parcourt l'espace des phases de la matière et excite l'ensemble des degrés de liberté accessibles, ceux-là même qui émergent de la collision de simples électrons ou protons dans les grands accélérateurs de particules. Mais, contrairement à une image trop galvaudée, ces derniers ne permettent pas de recréer in vitro les conditions vécues par l'univers aux premiers instants après le Big-Bang, puisqu'ils vont explorer le diagramme des phases de la matière à grand potentiel chimique, c'est-à-dire avec une énergie potentielle élevée, et non à haute température.

\section{Que faudrait-il pour avoir un mécanisme efficace de formation de la matière ?}

Nous vivons dans un monde matériel. Et pourtant, comme nous l'a appris P.A.M. Dirac, les lois de la mécanique quantique ne s'accommodent de celles de la relativité qu'en présence d'antimatière : à chaque particule de matière est associée une antiparticule de même masse, mais de charge opposée. Deux mondes miroirs, presque identiques en somme. Mais l'antimatière ayant une fâcheuse tendance à s'annihiler avec la matière pour donner quelques photons, se pose la question de comment nous, matière, avons-nous émergé du Big-Bang et dominé l'antimatière. L'hypothèse d'une asymétrie initiale, d'un léger surplus de matière sur l'antimatière à la fin du Big-Bang, est aussi peu séduisante qu'incompatible avec une phase inflationnaire dans l'expansion de l'univers (voir question 5), qui a pour effet de complètement diluer tout excès de matière. Reste alors à faire appel à un processus dynamique permettant de générer cette asymétrie de la matière sur l'antimatière : on parle de mécanisme de baryogenèse.

Fait remarquable, le modèle standard (voir encadré 2) possède a priori tous les ingrédients nécessaires pour générer cette asymétrie : phénomènes hors d'équilibre pour assurer que le taux de production de baryons n'est pas égal au taux de disparition de ces mêmes baryons, brisure de la symétrie dite $\mathrm{CP}^{(\mathrm{a})}$ entre matière et antimatière et, bien sûr, violation de la conservation du nombre de baryons. Si les interactions électromagnétiques et les 
interactions fortes sont invariantes sous la symétrie $\mathrm{CP}$, les interactions faibles, elles, ne le sont pas. Et ceci est dû, comme l'ont compris M. Kobayashi et T. Maskawa, au fait que l'électron n'est pas seul au monde, mais qu'il existe trois familles de leptons et surtout trois familles de quarks (encadré 2). Hélas, la plupart de ces quarks sont beaucoup plus légers que les médiateurs des interactions faibles, les bosons $\mathrm{W}$ et $\mathrm{Z}$, ce qui rend la violation de $\mathrm{CP}$ du modèle standard trop faible pour produire l'asymétrie matière-antimatière désirée. De nouvelles sources de violation de CP sont donc nécessaires. C'est certes une carence du modèle standard, mais surtout un précieux indice pour comprendre la structure de ses possibles extensions !

\section{Et la matière noire, qu'en sait-on ?}

Dans la plupart de ses manifestations astrophysiques, la matière noire est introduite comme masse manquante pour résoudre une inadéquation observée entre effets gravitationnels et bilan de matière visible sous forme d'étoiles, de gaz chaud, etc. Certains verront là le signal d'une nécessaire modification des lois de la gravitation (voir question 7). Le rôle de la matière noire pour expliquer les petites anisotropies de température du fond diffus cosmologique, le rayonnement fossile émanant du Big-Bang, est toutefois plus subtil. On ne sait rendre compte des détails de ces observations qu'au prix de l'introduction d'une composante de matière sans charge électrique et dotée de peu d'énergie cinétique au moment de la recombinaison, lorsque les électrons se lient aux noyaux atomiques. C'est précisément cette nouvelle composante de matière qui va permettre, dans le plasma baryonique encore ionisé, le développement d'instabilités gravitationnelles aux petites échelles. Les propriétés attendues des particules de matière noire se précisent ici un peu : leur masse doit être supérieure au $\mathrm{keV} / \mathrm{c}^{2}$ (soit $1 / 500$ de la masse de l'électron) ; leurs interactions entre elles et avec le reste de la matière (et en particulier avec les photons) doivent être très faibles, typiquement avec une section efficace avec les photons de moins de $10^{-30} \mathrm{~cm}^{2}$. À travers ces observations, la matière noire devient plus qu'une hypothèse de travail : c'est une

\section{Le modèle standard de physique des particules}

encadré 2

Le modèle standard décrit les particules élémentaires et leurs interactions (fig. E2). La matière ordinaire est faite d'électrons et de quarks up et down. Les quarks n'existent qu'au sein d'états liés, tels que le proton (assemblage de deux quarks up et un quark down), le neutron (un quark up et deux quarks down) ou les mésons (paires de quark et d'antiquark). Les quarks et les leptons sont regroupés au sein de trois familles: les quarks up et down, l'électron, ainsi qu'une particule très légère et de charge électrique nulle, le neutrino électronique, forment la première famille. Des particules aux caractéristiques similaires, mais avec des masses plus élevées, constituent les deux autres familles. En 1973, M. Kobayashi et T. Maskawa (prix Nobel en 2008) ont montré que l'existence de trois familles de fermions était une condition nécessaire pour une violation de la symétrie CP entre matière et antimatière. Le modèle standard gouverne aussi les interactions entre ces particules de matière, ces interactions étant propagées par des particules de force appelées bosons de jauge : le photon pour les forces électromagnétiques, les bosons $\mathrm{W}$ et $\mathrm{Z}$ pour les forces faibles, et les gluons pour les interactions fortes. Les forces faibles, contrairement aux forces électromagnétiques, ont une portée finie de l'ordre de $10^{-15} \mathrm{~m}$. Cette portée finie est une conséquence de la masse des $\mathrm{W}$ et $\mathrm{Z}$ de l'ordre de cent fois la masse du proton alors que le photon, lui, est une particule de masse nulle. Une description cohérente des masses de ces bosons de jauge fait appel au mécanisme de BroutEnglert-Higgs (prix Nobel en 2013). Un champ scalaire, c'est-à-dire un degré de liberté sans spin, bien connu sous le nom de boson de Higgs, joue le rôle de médiateur et assure que les masses des $W$ et $Z$, qui brisent les symétries de jauge, ne mènent à aucune pathologie à grande énergie [1]

Le boson de Higgs est-il l'ultime pièce manquante du modèle standard ? Du modèle standard, peut-être, mais certainement pas du grand puzzle de la structure intime des lois de la physique à des énergies de plus en plus élevées. On sait, depuis une vingtaine d'années, que les neutrinos sont eux aussi massifs. $\mathrm{Si}$, comme pour les autres fermions, ces masses sont elles aussi engendrées par le mécanisme de Higgs, alors de nouveaux neutrinos devraient exister. Contrairement à ceux déjà connus, ces nouveaux neutrinos n'auraient aucun couplage aux bosons $W$ et $Z$, et toujours sans charge électrique, ils n'interagiraient pas non plus avec les photons : on parle de « neutrinos stériles». D'autre part, tout laisse à penser que le monde devrait devenir supersymétrique à des énergies de l'ordre de quelques $\mathrm{TeV}$ : chaque particule du modèle standard serait alors reliée à une nouvelle particule de spin différent. Une alternative conçue par les physiciens théoriciens est que l'espace-temps compte de nouvelles dimensions repliées sur elles-mêmes. Et une façon de réconcilier les lois de la mécanique quantique avec celles de la gravité serait de cesser de voir les particules élémentaires comme des entités ponctuelles et sans structure mais, au contraire, de les considérer comme des vibrations d'objets étendus comme des cordes se propageant dans des espaces-temps à 10 ou 11 dimensions [2]. Des espaces-temps supersymétriques, bien sûr...

[1] G. Bernardi, Reflets de la physique 14 (2009) 10-14.

[2] M. Petropoulos, Reflets de la physique 22 (2010) 8-13, et 30 (2012) 12-16.

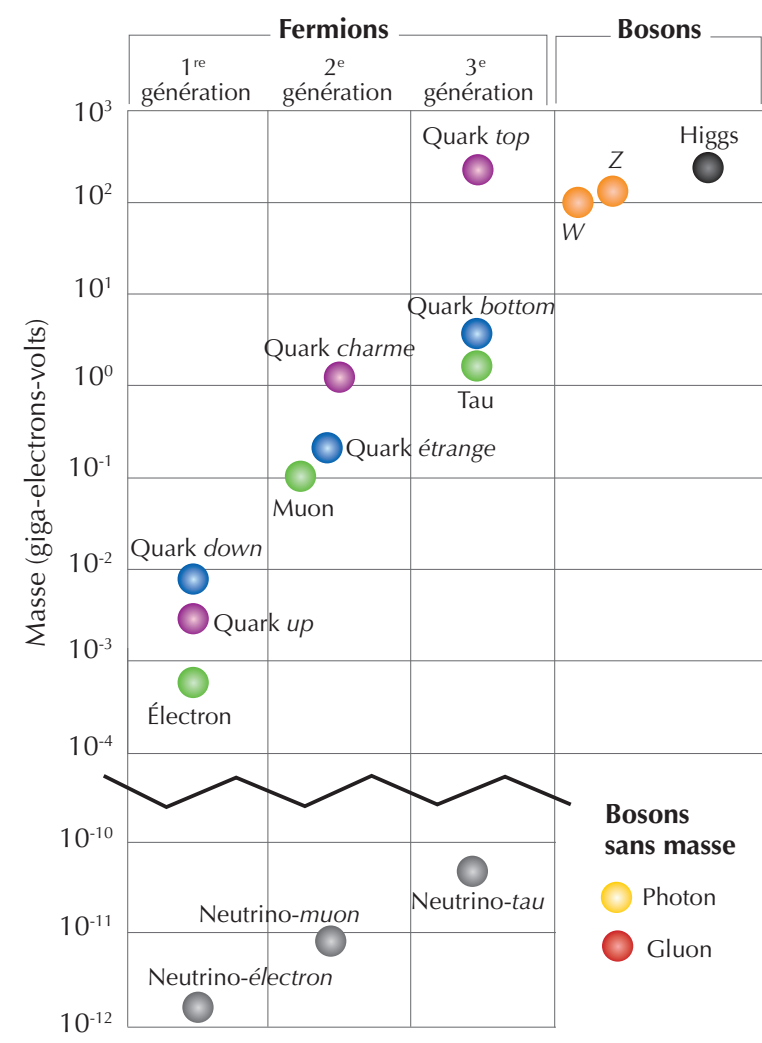

E2. Tableau des particules du modèle standard (en ordonnée, leurs masses). (D’après G. Kane, Scientific American 288 (2003) 68-75.) 
>>

véritable théorie prédictive, permettant de rendre compte avec économie d'observations maintenant très précises et très contraignantes.

Ce qu'il y a de troublant, c'est qu'aucune des particules du modèle standard de la physique des particules ne répond aux critères demandés. Ou, dit autrement, la cosmologie aurait ainsi identifié de nouveaux degrés de liberté non encore répertoriés en physique des hautes énergies, ni auprès des accélérateurs de particules, ni dans les gerbes de rayons cosmiques. Cette conclusion ravit bien sûr tous ceux des théoriciens qui ne voient dans le modèle standard qu'une simple théorie effective valable à basse énergie, et qu'il va falloir transcender et compléter lorsque les densités d'énergie deviennent de l'ordre de $10^{43} \mathrm{~J} / \mathrm{cm}^{3}$. Coïncidence heureuse ou preuve d'une réalité à confirmer, la matière noire trouve toute sa place dans la plupart des extensions du modèle standard rêvées par les chercheurs, comme la supersymétrie ou les espaces-temps avec des dimensions supplémentaires. Toutes ces théories prédisent l'existence de nouvelles particules répondant aux exigences énoncées. Qui plus est, si on cherche à estimer la section efficace de telles particules se formant au cours du refroidissement de l'univers, on calcule une densité résiduelle, c'est-à-dire le taux d'abondance de ces particules aujourd'hui, parfaitement compatible avec les estimations de la quantité de matière noire dans l'univers. Bref, il ne reste plus qu'à découvrir ces nouvelles particules : la traque est ouverte ${ }^{(b)}$.

\section{Et comment s'y prend-on pour découvrir la matière noire? Le LHC peut-il jouer un rôle dans cette recherche ?}

Dans le cadre de ce scénario de matière noire composée de WIMPs (pour "weakly interacting massive particles"), ce sont les interactions avec les particules ordinaires du modèle standard qui ont permis de maintenir les particules de matière noire en équilibre chimique et thermodynamique, jusqu'à ce que leur quantité présente dans l'univers se gèle quand leurs possibilités d'annihilation se raréfient avec l'expansion de l'univers. Ce sont ces mêmes interactions avec la matière ordinaire qui pourraient demain permettre de produire ces précieuses particules de matière noire lors de la collision de deux protons. En quelque sorte, le LHC, en mesurant les sections efficaces d'interaction de toutes ces particules, permettra de remonter à la densité de WIMPs et donc de "peser " la masse de l'univers.

Mais, est-ce là la seule façon d'observer la matière noire dans le laboratoire ? Pas tout à fait : il existe de nombreuses expériences, dites de détection directe, qui cherchent à mesurer le recul d'une particule ordinaire lors d'un choc avec une particule de matière noire provenant de rayons cosmiques (voir par exemple la description de l'expérience Edelweiss, dans le Bulletin de la SFP n¹55 (2006) 15). Ce sont des mesures délicates, car il faut s'affranchir de tout choc ordinaire parasite. Mais ces expériences ont maintenant atteint la sensibilité nécessaire pour corroborer les scénarios les plus en vogue sur la matière noire.

Et, si d'aventure tous ces scénarios se trouvaient mis à mal par les résultats expérimentaux, nous aurions encore beaucoup à apprendre du LHC, par exemple grâce à la découverte d'un axion, responsable de la non-violation de la symétrie CP pour les interactions fortes, ou d'un gravitino, le partenaire supersymétrique du graviton d'Einstein, deux particules dont les interactions avec la matière ordinaire sont si ténues qu'elles n'ont jamais pu être en équilibre thermodynamique. Un axion sous l'effet d'un champ magnétique, peut toutefois se transformer en photon, et vice versa. Sous certaines conditions de brisure de la supersymétrie, le gravitino est la particule supersymétrique la plus légère, donc automatiquement stable (car il n'y a pas d'autres particules supersymétriques plus légères en lesquelles elle peut se désintégrer), et devient de ce fait un excellent candidat à la matière noire. Son existence se manifesterait dans les détecteurs du LHC par un déficit d'énergie emportée au loin par ces gravitinos, dans lesquels toutes les particules supersymétriques produites finiraient leur vie.

5. L'inflation semble être devenue une pierre angulaire de la construction des modèles cosmologiques. Qu'en est-il ? Quel est le lien avec la physique des particules?

L'inflation, telle qu'on la définit aujourd'hui, est plus un paradigme qu'une véritable théorie complète et prédictive. Elle est basée sur l'introduction d'une phase d'expansion accélérée pendant l'histoire thermique de l'univers, qui permet d'expliquer la nature des fluctuations de la géométrie d'espace-temps à l'échelle des grandes structures de l'univers (les cosmologistes parlent de "fluctuations de métrique "). Ces fluctuations semblent a priori défier les lois de la causalité : par exemple, les variations de température du fond diffus cosmologique présentent des corrélations sur des échelles plus grandes que la distance causale de l'univers telle que calculée dans le contexte d'une cosmologie standard, c'est-à-dire une succession de phases d'expansions décélérées.

Il reste que les conditions physiques menant à une phase d'expansion accélérée ne sont pas naturelles, la matière sous sa forme ordinaire ne conduisant jamais à ce type de comportement. Le modèle d'inflation le plus simple emprunte à la physique des hautes énergies l'existence de particules dites scalaires, c'est-à-dire de spin 0 , comme le boson de Higgs récemment découvert au CERN. Même si certains modèles essaient de rendre le boson de Higgs responsable de la phase d'inflation, la plupart des modèles font appel à de nouvelles particules scalaires non encore identifiées par la physique des hautes énergies. Comme il a été établi dans les années 1980, les champs quantiques associés à ces nouvelles particules scalaires peuvent facilement être excités et placés dans une configuration conduisant à une phase d'expansion accélérée. De plus, pendant celle-ci, des fluctuations de métrique sont naturellement engendrées à des échelles devenues aujourd'hui « acausales » (c'est-à-dire trop grandes pour que chacune de ses parties puisse rester en relation causale) et qui seraient à l'origine des grandes structures de l'univers observées aujourd'hui. Il faut cependant noter que, pour élémentaire qu'il soit, le mécanisme mis en jeu fait appel à la théorie quantique des champs dans une situation sans équivalent en physique des hautes énergies ou en laboratoire.

De fait, l'inflation, par son économie de moyens, est devenue un paradigme extrêmement séduisant. Malheureusement, l'échelle d'énergie de cette phase est a priori très largement inaccessible aux expériences de laboratoire. En effet, le fait même que les fluctuations de métrique soient de l'ordre de $10^{-5}$ montre, par une simple analyse dimensionnelle, que les échelles d'énergie en jeu sont de l'ordre du millième de l'énergie de Planck ${ }^{(c)}$, soit 12 ordres de grandeur au-dessus de l'échelle d'énergie accessible au LHC, par exemple! 
6. On parle maintenant d'énergie noire. Est-ce une nouvelle matière noire, ou autre chose encore ?

L'énergie noire est la «force " responsable de l'accélération actuelle de l'expansion de l'univers, mise en évidence à la fin des années 1980 dans l'observation de la luminosité de supernovæ et corroborée depuis par de nombreuses autres observations cosmologiques. Le problème de l'énergie noire a plusieurs facettes. D'un point de vue de cosmologie observationnelle, l'introduction d'une constante cosmologique $\Lambda$ (un paramètre supplémentaire dans les équations de la relativité générale) comme nouvelle constante fondamentale de la théorie de la gravité, permet de réconcilier un ensemble maintenant très vaste d'observations et de contraintes. Celles-ci concernent aussi bien l'observation des anisotropies de température du fond diffus cosmologique, le comportement de la luminosité des supernovæ lointaines, le contenu en baryons des amas des galaxies, l'âge des plus vieilles étoiles ainsi que l'amplitude des fluctuations de densité de matière observées dans l'univers local. La question qui se pose légitimement est de savoir si ce nouveau paramètre correspond effectivement à une nouvelle constante de la nature ou au contraire à un objet dynamique, donc à un nouveau degré de liberté. Les deux hypothèses sont tout aussi inconfortables.

Si la théorie de la gravité admet deux constantes fondamentales, $G$ la constante de Newton et donc $\Lambda$, alors cette théorie introduit une nouvelle échelle d'action, $\mathrm{c} /(\Lambda \mathrm{G}) \approx 10^{87} \mathrm{~J} . \mathrm{s}$, qui n'a rien à voir (en tout cas numériquement) avec l'action élémentaire $h$ de la mécanique quantique, $h \approx 6,610^{-34} \mathrm{~J}$.s. Ainsi, si on voulait rendre compte de cette densité d'énergie comme étant celle des fluctuations quantiques des champs dans le vide, on aboutirait à une incompatibilité de 120 ordres de grandeur! $\mathrm{Si}$, au contraire, cet effet a pour origine un nouveau fluide, un nouveau degré de liberté, alors il peut être associé à une échelle d'énergie, disons la masse des particules qui lui seraient associées, qui serait de l'ordre de $10^{-3} \mathrm{eV} / \mathrm{c}^{2}$, ce qui est extrêmement bas par rapport aux échelles de masse habituelles de la physique des particules. Il reste que ce serait un fluide tout à fait nouveau, qui ne se comporte pas comme un gaz de particules - il a une pression effective négative ! - qu'on ne peut ni mettre en boîte ni détecter en laboratoire. On comprend le scepticisme des théoriciens. Pour autant, on dispose de moyens d'investigation : l'accélération actuelle de l'expansion de l'univers est un phénomène récent, qui a un effet sur la croissance de ses grandes structures. La recherche de signatures d'un comportement dynamique associé à l'énergie noire est tout l'enjeu du projet Euclid récemment sélectionné par l'ESA.

L'énergie noire et, dans une moindre mesure, la matière noire ont en commun de mettre en question les lois de la gravité aux distances cosmologiques. Ce qui est interprété comme leurs manifestations pourrait être le signal, non pas de nouveaux degrés de liberté dans l'espace des phases de la matière, mais d'une nouvelle dynamique.

\section{La physique des particules auprès} des accélérateurs permettrait-elle de mieux comprendre la gravité ?

La gravitation est fondamentalement différente des autres interactions fondamentales. Les forces électromagnétiques, faibles et fortes qui occupent la physique des particules sont toutes décrites par l'échange de particules de spin 1 (voir encadré 2). Le graviton de la relativité générale serait, lui, une particule de spin 2 qui offre moins de liberté et rend la théorie essentiellement unique ${ }^{(d)}$ et sans autre paramètre à ajuster que la constante universelle de gravitation. Mais la différence est aussi quantitative : ainsi, l'attraction gravitationnelle entre deux protons est de 42 ordres de grandeur plus faible que leur répulsion électrique. On serait donc naturellement amenés à conclure que des mesures précises au quarantième chiffre après la virgule vont être nécessaires pour nous renseigner sur la gravité.

Mais cette conclusion repose sur une hypothèse forte, à savoir que les lois de la gravitation telles que nous les connaissons depuis Einstein resteraient inchangées, et qu'elles s'appliquent aussi bien aux superamas de galaxies distants de plusieurs mégaparsecs, soit quelque $10^{23} \mathrm{~m}$, qu'aux quarks distants, eux, de seulement $10^{-18} \mathrm{~m}$ au sein des protons et neutrons. En fait, notre connaissance empirique de la gravité se cantonne à des distances comprises entre $10^{13} \mathrm{~m}$ (peu ou prou la taille du système solaire) et $10^{-4} \mathrm{~m}$. Cette constatation a conduit certains théoriciens à postuler qu'il pourrait exister des dimensions supplémentaires d'espace avec une taille de l'ordre de la centaine de microns, dans lesquelles seuls les gravitons seraient libres de se propager. Le comportement des interactions gravitationnelles à petite distance s'en trouverait alors profondément bouleversé et pourrait même devenir quantique à des distances de l'ordre de $10^{-19} \mathrm{~m}$, domaine cette fois-ci parfaitement accessible au LHC. Et si cette idée est si séduisante, c'est parce qu'elle apporte aussi une solution au problème de la stabilité de la physique des particules, en permettant de contrôler les corrections quantiques à la masse du boson de Higgs, un des grands problèmes qui occupent les théoriciens des hautes énergies.

\section{En conclusion, quelles sont les perspectives?}

Deux des grandes aventures intellectuelles des $20^{\mathrm{e}}$ et $21^{\mathrm{e}}$ siècles, l'écriture d'une histoire de l'univers et celle de l'inventaire des particules et des interactions élémentaires, se sont continuellement épaulées et enrichies l'une l'autre. Nous sommes arrivés une fois de plus à une croisée des chemins. Mécanisme d'inflation, matière noire, énergie noire sont autant d'ingrédients mal identifiés de notre recette cosmique, mais de quelque côté que viennent les premiers éléments de réponse, ils contribueront à enrichir notre palette de saveurs.

(a) La symétrie CP correspond à l'invariance d'une interaction par conjugaison de charge, transformation d'une particule en son antiparticule, et renversement de parité simultanés. Cette symétrie est brisée en présence de processus qui ne se passent pas de la même façon avec des particules et avec les antiparticules correspondantes.

(b) Une solution astrophysique au problème de la matière noire n'est pas non plus à exclure, comme par exemple une abondance de minitrous noirs, voire, pourquoi pas, des monopôles magnétiques dont l'existence a été prédite par P. Dirac en 1931, mais n'a jamais été confirmée expérimentalement.

(c) Si elle devait être confirmée, la détection récente par l'expérience Bicep 2 (voir http://arxiv.org/ abs/1403.3985) d'un fond d'ondes gravitationnelles au moment de la recombinaison est un résultat crucial. Elle permet de mesurer directement la densité d'énergie de l'univers pendant la phase d'inflation.

(d) Par exemple, on ne sait pas construire de théories avec plusieurs particules de spin 2 en interaction.

\section{En savoir plus}

- F. Bernardeau, E. Klein, S. Laplace, M. Spiro et S. Huet, La physique des infinis, Collection 360, La ville brûle (2013).

- G. Cohen-Tannoudji et M. Spiro, Le boson et le chapeau mexicain, collection Folio Essai, Gallimard (2013).

- C. Grojean et L. Vacavant, À la recherche du Boson de Higgs, Collection Librio Mémo, J’ai lu (2013). 